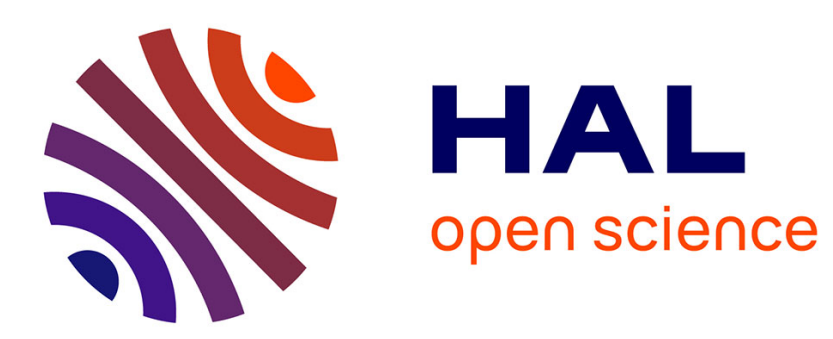

\title{
Electron stimulated desorption of solid argon via exciton creation
}

\author{
F. Coletti, J.M. Debever, G. Zimmerer
}

\section{To cite this version:}

F. Coletti, J.M. Debever, G. Zimmerer. Electron stimulated desorption of solid argon via exciton creation. Journal de Physique Lettres, 1984, 45 (9), pp.467-473. 10.1051/jphyslet:01984004509046700 . jpa-00232370

\section{HAL Id: jpa-00232370 https://hal.science/jpa-00232370}

Submitted on 1 Jan 1984

HAL is a multi-disciplinary open access archive for the deposit and dissemination of scientific research documents, whether they are published or not. The documents may come from teaching and research institutions in France or abroad, or from public or private research centers.
L'archive ouverte pluridisciplinaire HAL, est destinée au dépôt et à la diffusion de documents scientifiques de niveau recherche, publiés ou non, émanant des établissements d'enseignement et de recherche français ou étrangers, des laboratoires publics ou privés. 
Classification

Physics Abstracts

$78.60 \mathrm{H}-73.60 \mathrm{H}-68.45 \mathrm{D}$

\title{
Electron stimulated desorption of solid argon via exciton creation
}

\author{
F. Coletti, J. M. Debever and G. Zimmerer $\left(^{*}\right)$ \\ Groupe de Physique des Etats Condensés (**), Faculté des Sciences de Luminy, Case 901, \\ 70, route Léon-Lachamp, 13288 Marseille Cedex 9, France
}

(Reçu le 19 septembre 1983, révisé le 27 février 1984, accepté le 29 février 1984)

\begin{abstract}
Résumé. - Nous avons fait une étude comparée de la luminescence intrinsèque de l'argon solide et de la désorption du cristal excité par des électrons lents (qq. $\mathrm{eV}$ à $100 \mathrm{eV})$. Ces deux phénomènes présentent, pour une même énergie des électrons incidents, un seuil très marqué dû à la création d'excitons. Le taux de désorption est proportionnel à la densité d'excitons à la surface du cristal. Le caractère de « surface » de plusieurs raies de luminescence est établi. Le mécanisme de la désorption correspond à celui de la formation d'une cavité entourant l'exciton autopiégé dans l'argon solide.
\end{abstract}

\begin{abstract}
The correlation between the intrinsic luminescence of solid argon excited by slow electrons (a few eV to $100 \mathrm{eV}$ ) and desorption was studied. Related to exciton creation, a sharp threshold of desorption and of luminescence is observed at the same energy of the impinging electrons. The desorption rate is proportional to the exciton density at the surface. The surface nature of different luminescence bands is established. The mechanism of desorption corresponds to the mechanism of cavity formation around self-trapped excitons in solid argon.
\end{abstract}

\section{Introduction.}

Electron or photon stimulated desorption of ions and neutral atoms from crystals or adsorbed atom layers on metal surfaces is a problem of great current interest. More generally, the influence of the electronic excitation of a crystal on its cohesive energy is today an active field of research. Recently, Jennison and Emin [1, 2] pointed out that the localization of an electronic excitation at the surface of the crystal is an essential condition to stimulate desorption processes via primary electronic excitation. They especially pointed out that excitons may be involved in the desorption of neutral atoms.

Rare gas solids (RGS) have simultaneously weak cohesive energy, characteristic for Van der Waals forces, strong excitonic excitations at the onset of absorption in the vacuum ultraviolet spectral range, and strong exciton-phonon interaction which leads to rapid autolocalization (self-trapping) of excitons [3]. They therefore fulfil the requirement of localization of the electronic excitation for exciton stimulated desorption. Moreover, due to several luminescence studies on RGS, the properties of the self-trapped excitons are understood in detail, so that luminescence can easily be used as a probe for autolocalizing electronic excitations in these materials.

$\left(^{*}\right)$ On leave from II. Inst. für Experimentalphysik, Universität Hamburg, Hamburg, F.R.G.

$\left({ }^{* *}\right)$ ERA-CNRS 070373. 
Already ten years ago, Farrel et al. reported on RGS desorption stimulated by low energy electrons during LEED experiments [4]. They conclusively excluded thermal heating or sputtering processes as a source of desorption under their specific experimental conditions. They suggested that excitons may play a rôle in the desorption process, however without any direct experimental proof.

Low energy electron bombardement is also an efficient technique to excite the intrinsic luminescence of RGS (cathodoluminescence). It seemed to us therefore achievable as well as a worthwhile goal to look for an eventual correlation between desorption and luminescence of RGS under low energy electron bombardement. It turned out that solid argon is an especially good candidate because the luminescence spectra contain certain luminescence bands which are related to self-trapping of excitons at the surface of the sample.

\section{Techniques and results of cathodoluminescence of solid argon.}

The experimental set-up for cathodoluminescence measurements on RGS was described in detail elsewhere [5]. Briefly, pure argon gas is introduced into the vacuum chamber initially evacuated below $5 \times 10^{-9}$ torr. The gas condenses on a temperature regulated quasimonocrystal of graphite $\left({ }^{1}\right)$. The thickness is controlled by adjusting the exposure (pressure $\times$ time) assuming a sticking coefficient of one up to $27 \mathrm{~K}$. The impinging electrons are emitted by a hot tungsten filament about $1 \mathrm{~mm}$ in front of the sample. The luminescence is analysed by a VUV monochromator. Luminescence spectra are recorded under fixed electron energy and electron current. In a cathodoluminescence excitation spectrum (CE spectrum), the intensity of a spectrally selected luminescence band is recorded as a function of the incident electron energy $E$. The current $I$ flowing through the sample is measured simultaneously.

The samples were condensed at $27 \mathrm{~K}$ (slightly below the sublimation temperature) to ensure good crystalline quality. Most of the measurements were made at the same temperature. The results were also established at lower temperatures $(8 \mathrm{~K})$.

The fluorescence of solid argon was studied extensively and reviewed by Fugol [3]. Figure 1 shows a typical spectrum of solid argon excited by $5 \mathrm{keV}$ electrons obtained a few years ago [7].

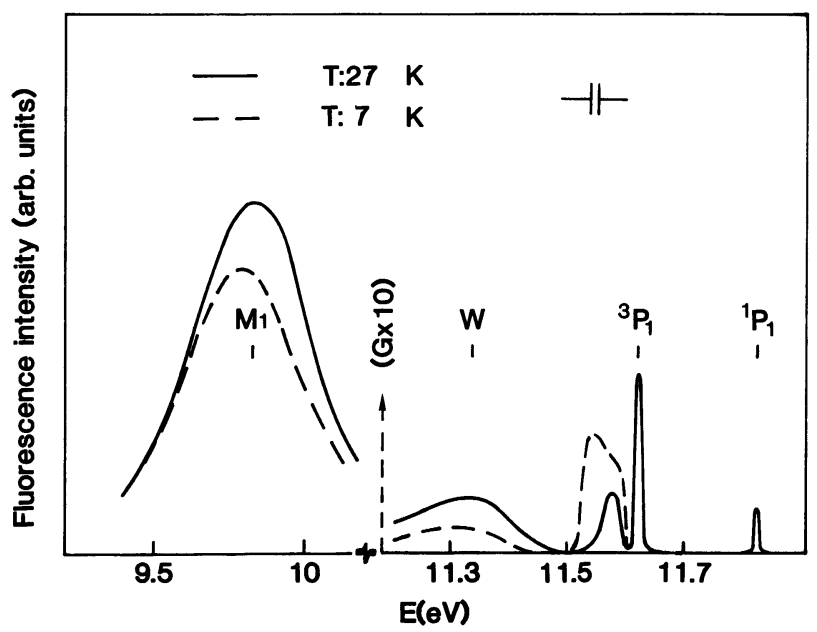

Fig. 1. - Cathodoluminescence spectra of solid argon $(d=1 \mathrm{~mm}$, electron energy $E=5 \mathrm{keV})$.

( $\left.{ }^{1}\right)$ Graphite was chosen because on this substrate epitaxial growth is possible under the experimental conditions used here [6]. An in situ analysis of the crystal structure during our experiments was not possible. 
Almost the same structures are observed as by Fugol, but with a better resolution (about $2 \AA$ ) :

(i) Quasi-atomic luminescence lines $\left(b^{0}\right.$ and $b^{1}$ of Fugol) which are resolved in two very sharp lines (resolution limited) at the same energy as the ${ }^{3} \mathrm{P}_{1}(11.62 \mathrm{eV})$ and the ${ }^{1} \mathbf{P}_{1}(11.82 \mathrm{eV})$ atomic levels, and two temperature sensitive broader lines not well resolved, at $11.55 \mathrm{eV}$ and $11.58 \mathrm{eV}$. These luminescence features are generally ascribed to the atomic-type self-trapped exciton (excited atom, $\mathrm{Ar}^{*}$, in the deformed lattice) [3, 7].

(ii) Luminescence of the molecular-type self-trapped exciton $\left(\operatorname{Ar}_{2}^{*}\right)$ with the $\mathrm{W}$ band at $11.34 \mathrm{eV}$ and the $M_{1}$ band at $9.8 \mathrm{eV}$.

When the electron energy is reduced to, e.g., $20 \mathrm{eV}$, the ${ }^{3} \mathrm{P}_{1}$ and $\mathrm{W}$ lines remain the same, the ${ }^{1} \mathrm{P}_{1}$ line is almost undetectable. The quasi-atomic lines at $11.55 \mathrm{eV}$ and $11.58 \mathrm{eV}$ are only visible on thick samples ( $d \gtrsim 300 \AA$ ), and the $\mathrm{M}_{1}$ band is less intense than the W band.

With optical excitation and with surface coverage experiments it was shown recently, that the $W$ band and the emission of atomic-type self-trapped excitons mainly stems from luminescence centres at the surface of the sample, whereas the $M_{1}$ band is emitted by centres in the bulk [8]. As the penetration depth of low energy electrons above the onset of inelastic electron-electron scattering is very small ( $\sim 5 \AA ; E \gtrsim 24 \mathrm{eV}$ ) [9], it is quite natural, that the surface sensitive features are enhanced compared with the luminescence features of the bulk.

The cathodoluminescence spectra are in good agreement with photoluminescence spectra [8]. Thus it is clearly ruled out that the light emission observed in our experiment is due to inverse photoemission.

Figure 2 shows a typical $\mathrm{CE}$ spectrum taken at $27 \mathrm{~K}$ on the $\mathrm{W}$ band and the corresponding $I(E)$ curve (sample thickness $150 \AA$ ). Similar results were also obtained for the ${ }^{3} \mathrm{P}_{1}$ line and the $\mathrm{M}_{1}$ band. The CE spectrum yields a sharp onset at $E_{1}=14 \mathrm{eV}$ and a second increase at $E_{2}=26 \mathrm{eV}$. We have given previously our interpretation of these marked structures [5]. The threshold energy $E_{1}$ for luminescence excitation is associated with the creation of an exciton by inelastic scattering of the incident electron to the bottom of the conduction band. For $E>E_{2}$, the incident electron has an energy large enough to create two excitons in adjacent inelastic scattering events. The difference between $E_{1}=14 \mathrm{eV}$ and the $\Gamma(3 / 2) n=1$ exciton of argon at $12.2 \mathrm{eV}$ [3] measures the momentum dispersion of the exciton band.

The luminescence intensities of the different bands are proportional to the incident current.

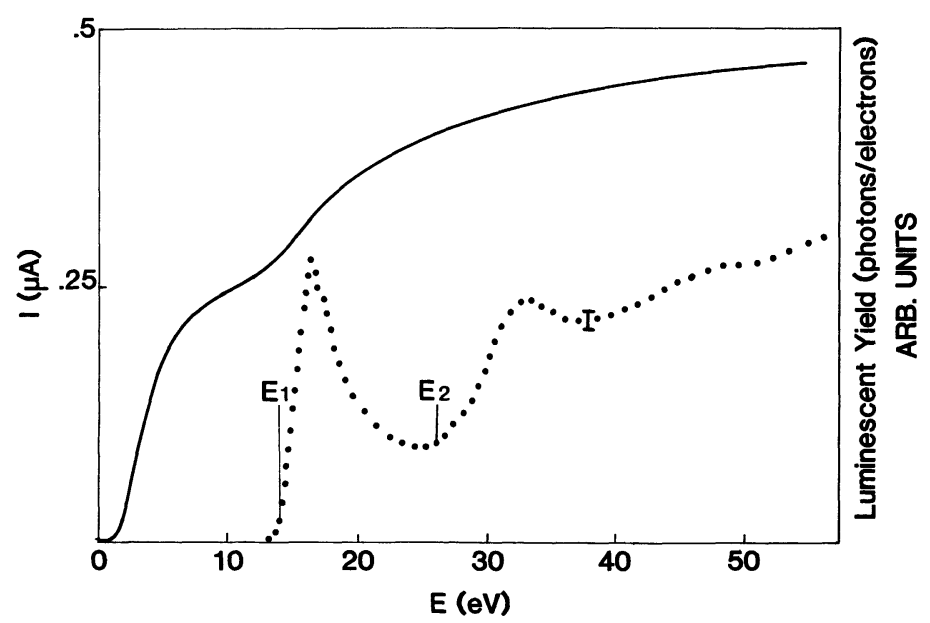

Fig. 2. - Points : Cathodoluminescence excitation spectrum of solid argon $(d=150 \AA, T=27 \mathrm{~K})$. The energy $E$ of the incident electrons is measured from the bottom of the conduction band. Full line : electron current $I$ through the sample versus $E$. 
During the measurements of a luminescence or a CE spectrum, the current must be kept low $(I \lesssim 0.1 \mu \mathrm{A})$ in order to minimize two phenomena, namely (i) desorption of argon (Sect. 3), and (ii) space charge effects.

Figure 3 displays the intensity of the $\mathrm{W}$ band $(E=24 \mathrm{eV}, T=27 \mathrm{~K}, I=0.5 \mu \mathrm{A})$ as a function of sample thickness $d$. The shape of this curve does not depend on $E$ and saturates only for $d \approx$ $4000 \AA$. This is a surprising result. When $E$ increases from $E_{1}$ to $24 \mathrm{eV}$ (first minimum of the CE spectrum), the penetration depth of the incident electrons decreases from a few thousand $\AA$ to a few $\AA$, as is known from Schwentner's photoemission experiments [9]. Then the primary excitation takes place in a thin layer at the surface. Nevertheless, the influence of the substrate (quenching) on the stationary state concentration of excitons which decay radiatively, diminishes only for $d \gtrsim 4000 \AA$. We must therefore postulate an exciton diffusion length of the order of $1000 \AA$ which is considerably larger than the already published values [10]. We want to point out, however, that the diffusion length may depend on the size of the crystallites of the evaporated layers. On the graphite substrate, at an elevated temperature of condensation, we expect monocrystalline epitactical islands [6], so that our result and the result of reference [10] may not be contradictory one to each other.

\section{Manifestation of desorption and measurement of desorption yield curves.}

The desorption of solid argon under low energy electron bombardement with an appropriate electron energy and current manifests itself — as in the work of Farrel et al. [4] — in a simple way. The samples disappear at a rate of $\sim 0.5 \AA / \mathrm{s}(E=15 \mathrm{eV}, I=5 \mu \mathrm{A})$. In a thickness range below $\sim 4000 \AA$, the desorption can be monitored by a continuous decrease of luminescence intensity of, e.g., the $\mathrm{W}$ band (see Fig. 3). The desorption phenomenon imposes severe limitations on the measurement of luminescence and CE spectra. Only for currents below $\sim 0.1 \mu \mathrm{A}$, the intensity variation between two successive measurements of luminescence spectra is negligible. This is the reason for the restrictions mentioned in section 2 .

The most important qualitative result concerning desorption is the following. If we use electrons with an energy $E<E_{1}$ (threshold of luminescence in the CE spectrum), no desorption is observed.

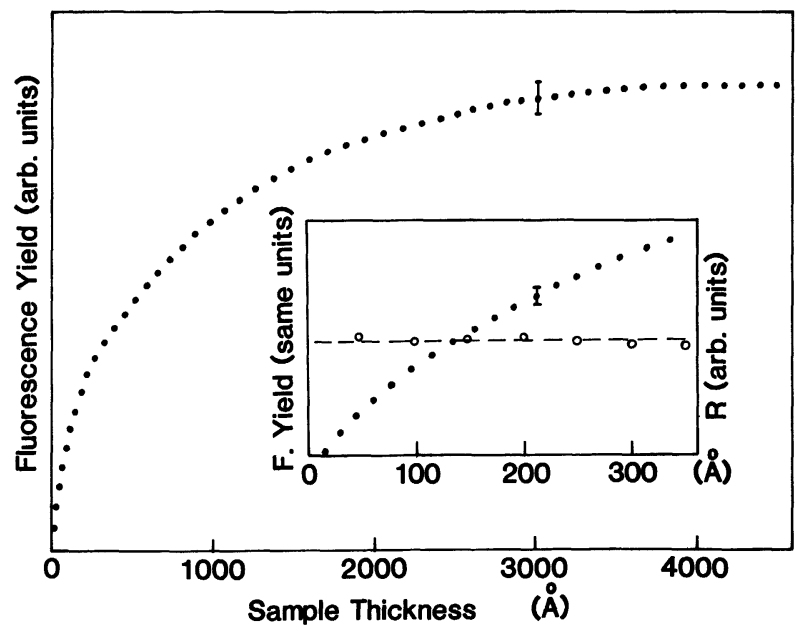

Fig. 3. - Luminescence intensity versus sample thickness of solid argon $(T=27 \mathrm{~K}, I=0.5 \mu \mathrm{A}, E=$ $24 \mathrm{eV}$ ). The insert shows a magnification of the curve at the onset (points) and the ratio $R$ between the stabilization pressure and the luminescence intensity. 
The luminescence intensity measured with $E>E_{1}$ is independent from electron bombardement with $E<E_{1}$. If electrons with $E>E_{1}$ are used, desorption occurs as discussed above. Slight modifications of this result by thermal heating effects are discussed below.

The qualitative result already clearly shows that there exists a correlation between primary electronic excitation of argon and desorption. In order to obtain quantitative results we had to look for conditions under which the thickness of the desorbing sample could be kept constant. This was possible adjusting the partial pressure of argon in the sample chamber in a way that the desorption losses were balanced by condensation. For a given electron energy $E$ and a current $I$, the appropriate argon partial pressure (stabilization pressure) was found by stabilizing the photon counting rate of a luminescence band as measured in the CE spectrum (desorption negligible) and multiplied by an appropriate factor taking into account the higher electron current. This procedure is only allowed, because the luminescence intensity as a function of thickness (Fig. 3) does not depend on the electron energy (relative shape). The stabilization procedure was done point by point because desorption is a strongly varying function of $E$.

Figure 4 shows the stabilization pressure as a function of incident electron energy $E$ for a fixed electron current $I=5 \mu \mathrm{A}$ (open circles). The most striking result is the threshold of desorption which is found at the same electron energy $E_{1}$ as the threshold of luminescence. This clearly shows that there exists a desorption process which takes place only after primary excitation of an exciton. In figure 4 we have included a $\mathrm{CE}$ spectrum of the ${ }^{3} \mathrm{P}_{1}$ line (full points) which was measured under the same experimental conditions. Over a wide range of electron energy, the stabilization pressure (desorption rate) and luminescence intensity are proportional one to each other. Concerning the ${ }^{3} \mathrm{P}_{1}$ line, the $\mathrm{CE}$ spectrum itself depends on stabilization pressure. In the low current limit, without stabilization pressure, it has a shape similar to the curve shown in figure 2. The CE spectrum of the $\mathrm{W}$ band, however, does not change with stabilization pressure.

An important experimental result of this study is the variation of the stabilization pressure as a function of sample thickness. The insert of figure 3 shows the ratio of the stabilization pressure and the $\mathrm{W}$ line intensity for fixed incident electron energy $E=24 \mathrm{eV}$ (open circles). Within the experimental uncertainty, this ratio is independent of thickness. Similar results were obtained with the ${ }^{3} \mathrm{P}_{1}$ line. At first sight this may be surprising but has a quite natural explanation. The electronic excitations leading to desorption need to be localized at or near to the surface $[1,2]$. On the other hand, both the $\mathrm{W}$ band and the ${ }^{3} \mathrm{P}_{1}$ line are known to be correlated with excitons-trapping at the surface [8]. So, both quantities must show the same dependence on thickness.

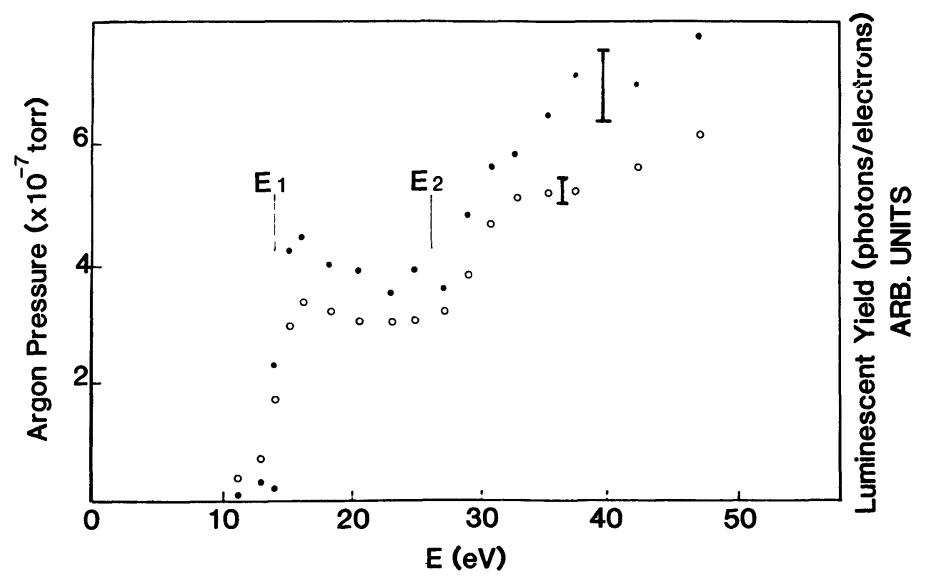

Fig. 4. - Circles : stabilization pressure of argon versus electron energy $E(T=27 \mathrm{~K}, d=150 \AA, I=$ $5 \mu \mathrm{A})$. Points : $\mathrm{CE}$ spectrum of the ${ }^{3} \mathrm{P}_{1}$ line obtained under the same conditions. 
We carefully checked that the stabilization pressure is proportional to the electron current (for fixed electron energy). As the luminescence itself is also proportional to the current and the ratio of both quantities is independent of the thickness, the desorption rate is proportional to the density of excitons at the surface.

Above $I \approx 5 \mu \mathrm{A}$, direct thermal heating effects are detectable and cause desorption also in the energy range $E<E_{1}$. But even at high currents $(10 \ldots 20 \mu \mathrm{A})$, the threshold at $E=E_{1}$ superimposed to the thermal desorption background is still a drastic effect. The thermal effect most probably occurs via the substrate because direct heating of RGS is inefficient. The inelastic scattering of low energy electrons on phonons is ineffective because in RGS (fcc-lattice) no optical phonons exist and the energies of acoustical phonons are below $\sim 5 \mathrm{meV}$ [3]. The electrons with $E<E_{1}$ therefore pass through the sample without heating [11]. Moreover, RGS are transparent from the far IR to the VUV so that they cannot absorb the radiation emitted by the tungsten filament. This was checked carefully.

\section{Mechanism of exciton stimulated desorption of solid argon.}

Though self-trapping of excitons in the bulk and at the surface of RGS is a well-known phenomenon, this is not necessarily a straightforward explanation of the exciton stimulated desorption process. Jennison and Emin pointed out that the problem of desorption is distinct from that of self-trapping on a surface [2]. Self-trapping causes deformation of the lattice. Desorption requires destruction of a bond.

We want to point out, however, a specific property of the light RGS. Due to the negative electron affinity of $\mathrm{Ne}$ and $\mathrm{Ar}$ [12], self-trapped excitons always repel the lattice and a cavity is formed around the localized excitation [3]. This cavity formation was convincingly demonstrated by Kanzaki and Suemoto [13] for solid neon. The diameter is given by the balance of different contributions to the total energy of the self-trapped exciton interacting with the lattice.

Recently, Kusmartsev and Rashba [14] calculated the energy of a self-trapped exciton inside a cavity of radius $R_{\mathrm{c}}$ including the following contributions : (i) Coulomb interaction of electron and atomic core, (ii) interaction of both electron and atomic core with the surrounding medium by mirror force, (iii) surface energy of the cavity, and (iv) elastic deformation energy of the crystal. In figure 5, we reproduce their result on the molecular-type self-trapped exciton, $\operatorname{Ar}_{2}^{*}$. If such a cavity could move towards the surface, it would be quite natural that the self-trapped exciton

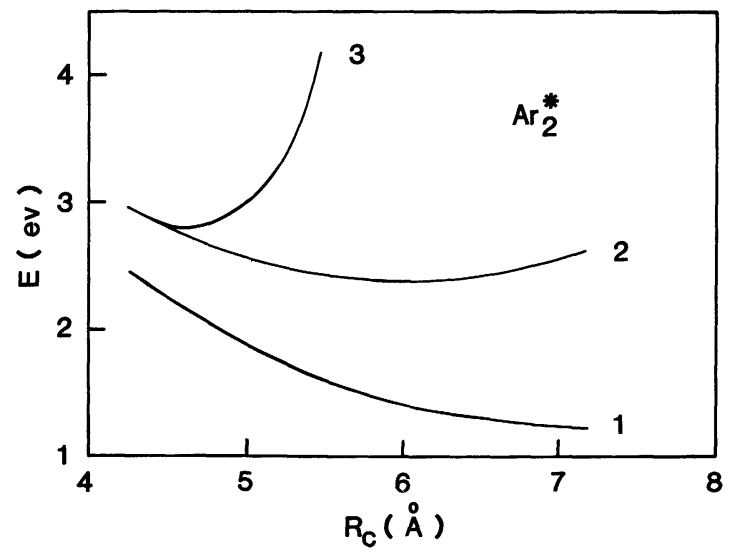

Fig. 5. - Energy of the molecular-type self-trapped exciton in argon as a function of cavity radius, $R_{\mathrm{c}}$. In curve 2 , the elastic energy is neglected. In curve 1 , the elastic energy and the surface energy are neglected. The results are taken from Ref. [14]. 
inside the cavity would be ejected from the surface thus minimizing the elastic strain of the crystal and the surface energy. This would correspond to the limit $R_{\mathrm{c}} \rightarrow \infty$ of the lowest curve in figure 5 which displays the total energy ignoring the surface energy and the elastic energy around the cavity. It seems therefore entirely reasonable that in the special case of the light RGS the self-trapping process at the surface directly leads to an ejection of the self-trapped exciton (either an excited atom or an excited molecule). Indeed, we could observe strong exciton enhanced desorption also for solid neon, but not for solid xenon. Concerning solid krypton, the effect is detectable but very weak.

There is direct experimental evidence for the proposed mechanism. The ${ }^{3} \mathrm{P}_{1}$ luminescence line coincides within the experimental error limit of $\pm 5 \mathrm{meV}$ with the position of the free atom ${ }^{3} \mathrm{P}_{1}$ line. Obviously we observe the radiative decay of such ejected excited atoms. Concerning the molecular-type self-trapped exciton, it was reported that the vibrational relaxation at the surface is slowed down by orders of magnitude compared with the bulk [8]. A quite natural explanation would be that these $\mathrm{Ar}_{2}^{*}$ molecules are ejected in high vibrational levels during the course of selftrapping at the surface. This mechanism of desorption via cavity formation would always lead to the ejection of excited neutral species.

We must take into account another possibility. As a consequence of the calculation of Kusmartsev and Rashba, the energetic position of the luminescence of self-trapped excitons in the solid and in the liquid phase (where the elastic strain is absent) should be quite different [14]. This can be concluded from the different energies of the minima of curve 1 (solid) and curve 2 (liquid) in figure 5. From the experimental point of view this is clearly not the case $[12,15]$. Kusmartsev and Rashba therefore propose, that the self-trapping process is accompanied by plastic deformation of the crystal (defect formation like pushing atoms to interstitial positions). Such a defect formation process during self-trapping at the surface could result in the ejection of a neutral ground state atom.

More insight into the desorption problem via electronic excitation of RGS could be obtained from model calculations of self-trapping at the surface including the possibility that either the excited species or ground state atoms can move out of the surface (this possibility is not included in calculations of self-trapping in two-dimensional systems [16]). This seems to us an achievable as well as worthwhile goal for future theoretical work.

\section{References}

[1] Jennison, D. R. and Emin, D., J. Vac. Sci. Technol. A 1 (1983) 1154.

[2] Jennison, D. R. and Emin, D., Phys. Rev. Lett. 51 (1983) 1390.

[3] Fugol', I. Ya., Adv. Phys. 27 (1978) 1.

[4] Farrel, H. H., Strongin, M. and Dickey, J. M., Phys. Rev. B 6 (1972) 4703.

[5] Coletti, F. and Debever, J. M., Solid State Commun. 47 (1983) 47.

[6] Seguin, J. L., Suzanne, J., Bienfait, M., Dash, J. G. and Venables, J. A., Phys. Rev. Lett. 51 (1983) 122 ;

SuZANNE, J., private communication.

[7] Coletti, F. and Bonnot, A. M., Chem. Phys. Lett. 55 (1978) 92.

[8] Roick, E., Gaethke, G., Zimmerer, G., Gürtler, P. and Woodruff, T. O., J. Phys. C., in press.

[9] Schwentner, N., Phys. Rev. B 15 (1976) 5490.

[10] Ophir, Z., Schwentner, N., Raz, B., Skibowski, M. and Jortner, J., J. Chem. Phys. 63 (1975) 1072.

[11] Sanche, L., Perluzzo, G., Bader, G. and Garon, L. G., J. Chem. Phys. 77 (1982) 3285.

[12] Jortner, J., Mayer, L., Rice, S. A. and Wilson, E. G., J. Chem. Phys. 42 (1965) 750.

[13] Suemoto, T. and Kanzaki, H., J. Phys. Soc. Jph 49 (1980) 1039.

[14] Kusmartsev, F. V. and Rashba, E. E., Czech. J. Phys. B 32 (1982) 54.

[15] Fugol', I. Ya., Savchenko, E. V. and Below, A. G., Fiz. Nizk. Temp. 1 (1975) 750.

[16] Toyozawa, Y. and Shinozuka, Y., J. Phys. Soc. Jpn 48 (1980) 472. 